\title{
Myocarditis combined with hypertrophic cardiomyopathy: a case report
}

\author{
Jing-Yue Wang, Yu-Shi Wang, Bo-Tao Shen \\ Department of Cardiology, The First Hospital of Jilin University, Changchun, China \\ Correspondence to: Yu-Shi Wang. Department of Cardiology, The First Hospital of Jilin University, No. 71 of Xinmin Street, Changchun 130021, \\ China. Email: yushi@jlu.edu.cn.
}

\begin{abstract}
Myocarditis can cause ventricular wall thickening due to myocardial edema. If the condition improves, the ventricular wall thickening should gradually decrease; a persistent thickening of the patient's ventricular wall indicates the coexistence of hypertrophic cardiomyopathy (HCM) and myocarditis. A 30-year-old man was referred to our hospital with continuous chest pain accompanied by profuse sweating. He suffered from fever for two days (the maximum body temperature: $38^{\circ} \mathrm{C}$ ) and the conditions improved following the use of antipyretics as self-administered medication before admission. Electrocardiogram exhibited ST-segment elevation in leads I and avL, and ST-T wave changes in leads II, III, avF, and V1-V6. Marked elevation of cardiac troponin I was found on laboratory testing. Respiratory tract infection testing showed negative results. A TORCH screen revealed positive herpes simplex virus (HSV), rubella virus (RV), and cytomegalovirus (CMV) IgG but all with negative IgM titer. Ultrasonic echocardiography showed thickness of the interventricular septum $(17 \mathrm{~mm})$ and diffuse left ventricular (LV) hypokinesia, without LV outflow tract obstruction. After consultation with the cardiology team, a diagnosis of myocarditis with HCM was made. Patients with myocarditis should be alerted to the possibility of HCM when there is persistent ventricular wall thickening.
\end{abstract}

Keywords: Hypertrophic cardiomyopathy (HCM); myocarditis; diffuse left ventricular hypokinesis (LV hypokinesis); interventricular septum; case report

Submitted Feb 08, 2021. Accepted for publication Apr 15, 2021.

doi: 10.21037/apm-21-359

View this article at: http://dx.doi.org/10.21037/apm-21-359

\section{Introduction}

Hypertrophic cardiomyopathy (HCM) is a complex and genetic cardiovascular disorder with an autosomal dominant pattern of inheritance that occurs with an estimated prevalence of $1 / 1,000$ in the general population and is characterized by a highly heterogeneous phenotype, unexplained left ventricular (LV) hypertrophy, cardiac fibrosis and myocyte disarray $(1,2)$. Myocarditis is an inflammatory disease of the cardiac muscle (myocardium) that is caused by different infectious and noninfectious triggers, including viral infections, bacterial infections, immune diseases, toxins, etc. Myocarditis can be acute, subacute, or chronic, and its signs and symptoms vary and include fever, chest pain, fatigue, shortness of breath, and arrhythmia (3). Previous reports have described thickening of the ventricular wall due to transient myocardial interstitial edema in patients with acute myocarditis $(\mathrm{AM})(4,5)$. However, reports of myocarditis in patients with HCM are rare. The present report presents a case of thickening of the ventricular wall a month post-AM, suggesting HCM was pre-existing. We present the following article in accordance with the CARE reporting checklist (available at http:// dx.doi.org/10.21037/apm-21-359).

\section{Case presentation}

A 30-year-old man presented to our hospital with continuous chest pain and a burning sensation below the breastbone accompanied by profuse sweating that 


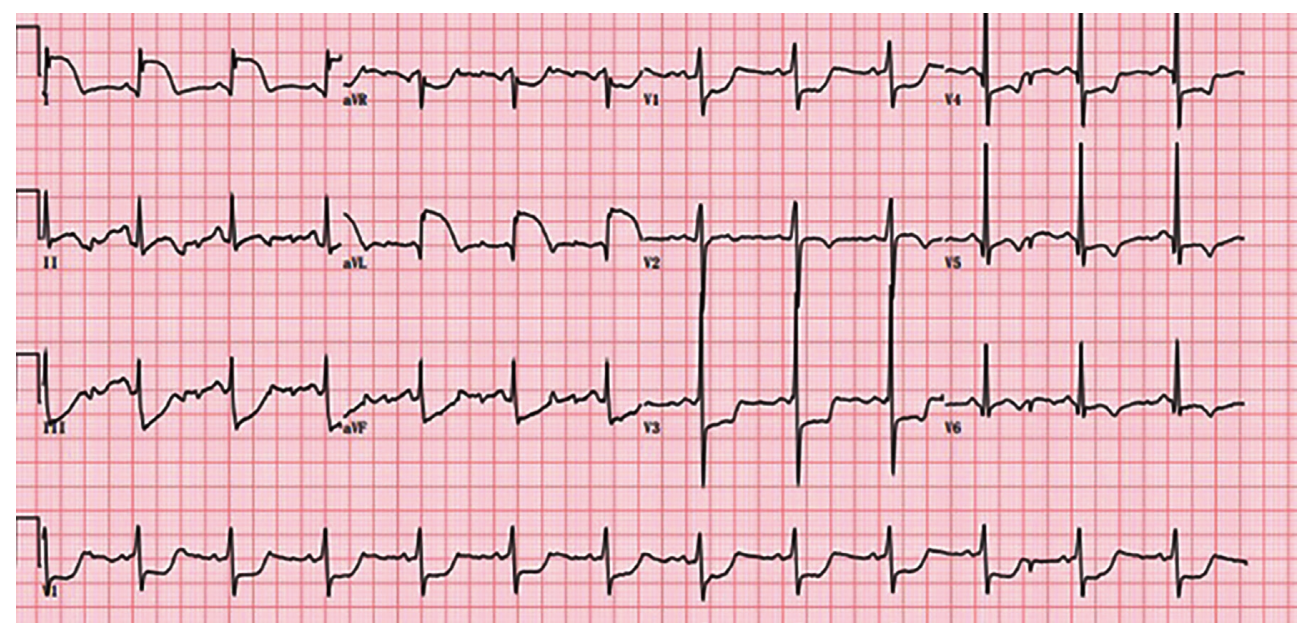

Figure 1 Electrocardiogram on admission showing ST-segment elevation in leads I and aVL, and ST-T wave changes in leads II, III, aVF, and V1-V6.

woke him at night. The patient reported to suffered from a fever for two days (maximum body temperature of $38{ }^{\circ} \mathrm{C}$ ), and conditions had improved following the use of antipyretics as self-administered medication. The patient denied tobacco smoking and alcohol consumption and had no significant past medical history. The man's parents and brother had a history of hypertension. Physical examination on admission was within the normal limits. The patient's vital signs were as follows: body temperature $36.5^{\circ} \mathrm{C}$, pulse rate of 78 beats $/ \mathrm{min}$ in a regular rhythm, respiratory rate of 16 breaths/min, and blood pressure of $111 / 63 \mathrm{mmHg}$. Lung auscultation showed normal and clear breath sounds and no rales, and cardiac auscultation revealed normal rate and rhythm and no murmurs. The skin and sclera were not yellow, and no cyanosis of the lips or lower limb edema was found. Electrocardiogram (ECG) exhibited ST-segment elevation in leads I and aVL and non-specific ST-T wave changes in leads II, III, aVF, and V1-V6 (Figure 1). The patient was admitted to the cardiac intensive care unit and was treated with the emergency green channel with $300 \mathrm{mg}$ bayaspirin and $180 \mathrm{mg}$ ticagrelor.

The patient underwent coronary angiography to further evaluate the ischemic etiology of the chest pain. Coronary angiography revealed no significant coronary artery stenosis with Grade 3 thrombolysis in myocardial infarction (TIMI) flow. The patient's laboratory data are shown in Table 1. White blood cell count, hemoglobin, and platelets were $8.18 \times 10^{9} / \mathrm{L}, 162 \mathrm{~g} / \mathrm{L}$ and $164 \times 10^{9} / \mathrm{L}$ on admission, respectively. Laboratory examination revealed marked elevation of cardiac troponin I (cTnI, $106 \mathrm{ng} / \mathrm{mL}$ ), creatine kinase-myocardial band (CKMB, $206.5 \mathrm{ng} / \mathrm{mL}$ ), and myoglobin (Myo, $502 \mathrm{ng} / \mathrm{mL}$ ). Laboratory findings also included an increase in the lactate dehydrogenase (LDH) level (1,254 U/L) and its alphahydroxybutyrate dehydrogenase of 1,106 U/L, an increase in aspartate aminotransferase (AST, 309.4 U/L) and alanine aminotransferase (ALT, 57.6 U/L), and an increase in serum total bilirubin (TBIL, $28.3 \mu \mathrm{mol} / \mathrm{L}$ ) and direct bilirubin (DBTL, $9.1 \mu \mathrm{mol} / \mathrm{L}$ ). Serum levels of thyroid-stimulating hormone (TSH, $6.850 \mathrm{uIU} / \mathrm{mL})$ and uric acid $(516 \mu \mathrm{mol} / \mathrm{L})$ were elevated. Free T3 and free T4 levels were within the normal range (Table 1). Evidence of a fluctuating fever and normal coronary arteries subverted the initial impression of coronary heart disease, and the findings were suggestive of myocarditis. Trimetazidine, coenzyme Q, vitamin C pills, sulbenicillin, meicelin, and spironolactone were administrated. Although the effect of spironolactone on myocarditis is not clear, it is recommended for patients with severe $\mathrm{LV}$ systolic dysfunction ( $\mathrm{EF}<35 \%$ ) or symptomatic heart failure. The bedside UCG showed that LVEF was $37 \%$, and the heart failure symptoms were obvious. All blood and sputum cultures were negative. Respiratory tract infection testing showed negative results. A TORCH screen revealed positive herpes simplex virus (HSV), rubella virus (RV), and cytomegalovirus (CMV) IgG but all with negative IgM titer. Intravenous drip of creatine phosphate sodium $(1 \mathrm{~g}, \mathrm{QD})$ and vidarabine $(0.5 \mathrm{~g}, \mathrm{q} 8 \mathrm{~h})$ was performed for 10 days.

Ultrasonic echocardiography (UCG) showed biatrial enlargement with LV systolic and diastolic dysfunction 
Table 1 The patient's laboratory data

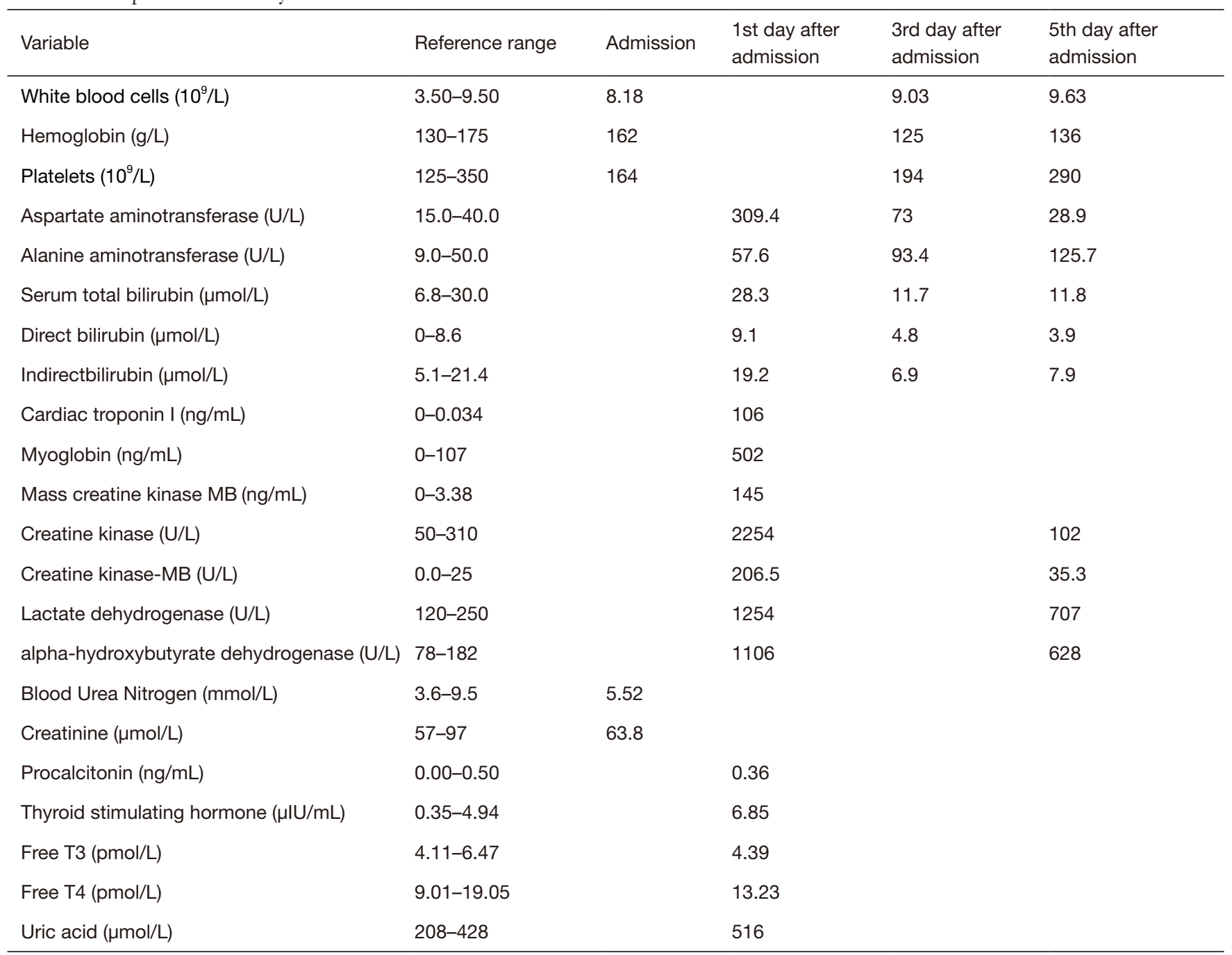

(LV ejection fraction 44\%), diffuse LV hypokinesia (Grade II), asymmetrical septal hypertrophy [ASH; thickness of the interventricular septum (IVS): $17 \mathrm{~mm}$ ], slightly elevated pulmonary artery pressure, and an end-diastolic diameter of $45 \mathrm{~mm}$. No LV outflow tract obstruction was observed. Moderate mitral and tricuspid regurgitation were noted, and a moderate pericardial effusion was observed (Figure 2). A cardiac magnetic resonance imaging (MRI) was ordered and further confirmed hypertrophy of the ventricular septum and anterior wall. $L V$ anterior wall is $9 \mathrm{~mm}$ thick in short axis end-diastole. The lowest wall is $8 \mathrm{~mm}$ thick, the lateral wall is $7 \mathrm{~mm}$ thick, and the ventricular septum is $22 \mathrm{~mm}$ thick at most. A betablocker (metoprolol succinate) was added on the third day after admission at a dose of $23.75 \mathrm{mg}$ (QD) and was increased to $47 \mathrm{mg}$ (QD) after MRI. Methylprednisolone was administered on the first day after admission and was stepped down gradually with improvement. The patient showed a significant improvement in symptoms, and laboratory findings were almost normalized. The patient reported that his family members also had interventricular septal hypertrophy detected by UCG. He and his family members were offered genetic testing, and the results showed variant of uncertain significance (VUS). A consultation with the cardiology team was conducted and collectively indicated a diagnosis of HCM with myocarditis. The patient was eventually discharged on day 12 of the hospital stay and was free of chest pain and without other symptoms prior to discharge. A post-discharge one-month follow-up visit showed that the patient was recovering 

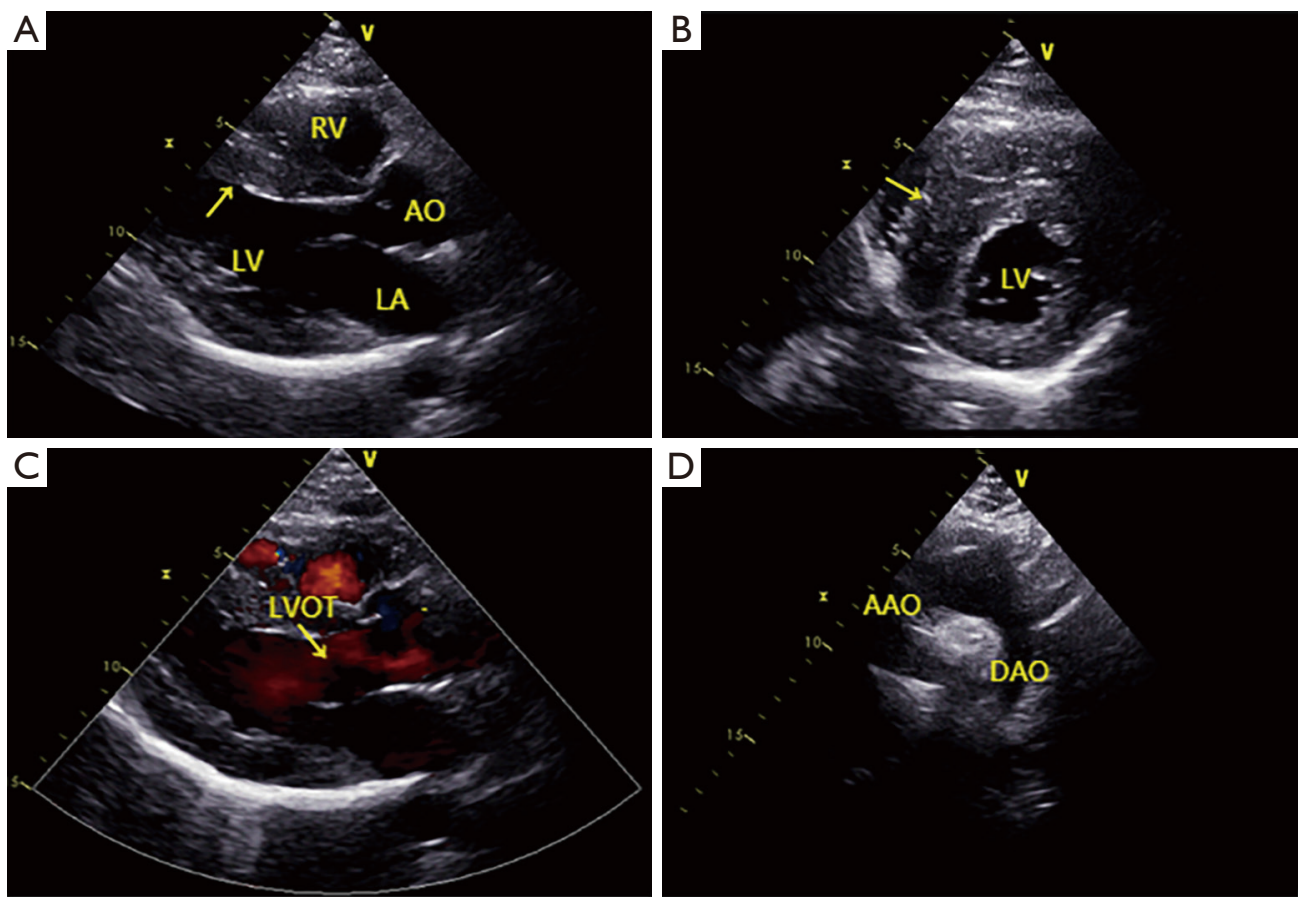

Figure 2 Ultrasonic echocardiography showed reduced left ventricular systolic function with asymmetric septal hypertrophy. (A) Twodimensional ultrasound: the parasternal LV long-axis view showed asymmetric left ventricular wall hypertrophy, mainly interventricular septum and thicker $17 \mathrm{~mm}$. (B) The parasternal short-axis view showed left ventricular wall was asymmetrically hypertrophic, mainly in the interventricular septum. (C) Color Doppler imaging showed that left ventricular outflow tract obstruction was not observed. (D) The long axis section of the great artery of the suprasternal fossa showed that there was no abnormality in the aortic arch and descending part. *Emergency green channel is a special green channel treatment mode for acute myocardial infarction in our hospital since 2009. It requires emergency doctors to establish simple cases, collect ECG and other auxiliary examinations, evaluate the indications of emergency percutaneous coronary intervention, exclude contraindications, and perform green channel intervention surgery. $\mathrm{V}$, ventricle; RV, right ventricle; AO, aorta; LV, left ventricle; LA, left atrium; LVOT, left ventricular outflow tract; AAO, ascending aorta; DAO, descending aorta.

well. However, UCG showed LV hypertrophy, as before. All procedures performed in studies involving human participants were in accordance with the ethical standards of the First Hospital of Jilin University and with the Helsinki Declaration (as revised in 2013). Written informed consent was obtained from the patient.

\section{Discussion}

It has been shown that AM can mimic HCM by causing an increase in $\mathrm{LV}$ wall thickness due to myocardial interstitial edema (5). The signs and symptoms of HCM can vary greatly among affected cases (6). Findings suggest that the diagnosis of HCM may be difficult in cases with AM. In the present study, we encountered a relatively rare case of patients with HCM combined with myocarditis.

There have been several reports of myocarditis combined with HCM (7-11). Takata et al. showed a case of sudden cardiac death from HCM and acute Fiedler's myocarditis (7). Another report indicated extensive left ventricular wall motion abnormalities and LV hypertrophy with extreme outflow obstruction in a 65-year-old man due to $\mathrm{AM}$ in HCM (11). In addition, Kusumoto et al. suggested that AM with $\mathrm{HCM}$ should be considered in patients presenting with cardiogenic shock due to left ventricle outflow obstruction and complete atrioventricular block (9). Our case was admitted to our clinic with complaints of chest pain and fever for two days before admission, and myocarditis combined with HCM was diagnosed after a thorough cardiac work-up and consultation with the cardiology team. The presence of virus (HSV, RV, CMV)-specific $\mathrm{IgG}$ in the absence of IgM indicated past infection at an undetermined time. In the present case, no LV outflow tract obstruction was observed by UCG. Diffuse LV hypokinesis 
was identified in the present study, which was suggestive of myocarditis (12), and there were symptoms of LV dysfunction. After resolution of the myocarditis, the diffuse hypokinesis of the left ventricle recovered.

The precise reason why edematous changes and myocardial damage due to myocarditis occurred mainly in the basal area of the IVS in HCM patients is unknown (9). Previous research has suggested that mechanical stress may enhance the damage to the myocardium caused by myocarditis because the basal area of the IVS is subjected to the stress of turbulent flow and high pressure induced by HCM (9).

HCM is a complex genetic cardiac disease. Around $40-60 \%$ of all cases of HCM are thought to be caused by a variety of autosomal dominant mutations in genes encoding sarcomeric proteins (e.g., myosin-binding protein $\mathrm{C}$, myosin light chain $3, \beta$-myosin heavy chain, cardiac troponin $\mathrm{I}$ and $\mathrm{T}$, and tropomyosin $\alpha-1$ chain) with a marked heterogeneity in clinical expression, natural history, and prognosis (13). It is well accepted that specific mutations remain unknown or cannot be detected in up to $30 \%$ of cases of HCM (14). Unfortunately, although the genetic testing was performed in the present study, we were unable to establish any significant genetic association of this case with HCM. After symptomatic treatment, anti-inflammation therapy, myocardial remodeling therapy, and methylprednisolone treatment, the patient was discharged and recovered well.

The present report presented a case of myocarditis accompanied with HCM. It is necessary to identify the associated abnormalities and evaluate the risk of myocarditis and accompanying HCM through a thorough cardiac workup to assist clinicians in decisions regarding therapy.

\section{Acknowledgments}

We would like to acknowledge the hard and dedicated work of all the staffs that implemented the intervention and evaluation components of the study.

Funding: None.

\section{Footnote}

Reporting Checklist: The authors have completed the CARE reporting checklist. Available at http://dx.doi.org/10.21037/ apm-21-359

Conflicts of Interest: All authors have completed the ICMJE uniform disclosure form (available at http://dx.doi. org/10.21037/apm-21-359). The authors have no conflicts of interest to declare.

Ethical Statement: The authors are accountable for all aspects of the work in ensuring that questions related to the accuracy or integrity of any part of the work are appropriately investigated and resolved. All procedures performed in studies involving human participants were in accordance with the ethical standards of The First Hospital of Jilin University (2020-654) and with the Helsinki Declaration (as revised in 2013). Written informed consent was obtained from the patient.

Open Access Statement: This is an Open Access article distributed in accordance with the Creative Commons Attribution-NonCommercial-NoDerivs 4.0 International License (CC BY-NC-ND 4.0), which permits the noncommercial replication and distribution of the article with the strict proviso that no changes or edits are made and the original work is properly cited (including links to both the formal publication through the relevant DOI and the license). See: https://creativecommons.org/licenses/by-nc-nd/4.0/.

\section{References}

1. Soler R, Méndez C, Rodríguez E, et al. Phenotypes of hypertrophic cardiomyopathy. An illustrative review of MRI findings. Insights Imaging 2018;9:1007-20.

2. Semsarian C. Update on the Diagnosis and Management of Hypertrophic Cardiomyopathy. Heart Lung Circ 2018;27:276-9.

3. Fung G, Luo H, Qiu Y, et al. Myocarditis. Circ Res 2016;118:496-514.

4. Morimoto S, Kato S, Hiramitsu S, et al. Role of myocardial interstitial edema in conduction disturbances in acute myocarditis. Heart Vessels 2006;21:356-60.

5. Hiramitsu S, Morimoto S, Kato S, et al. Transient ventricular wall thickening in acute myocarditis: a serial echocardiographic and histopathologic study. Jpn Circ J 2001;65:863-6.

6. Young L, Smedira NG, Tower-Rader A, et al. Hypertrophic cardiomyopathy: A complex disease. Cleve Clin J Med 2018;85:399-411.

7. Takata Y, Teraoka K, Abe M, et al. Sudden cardiac death from hypertrophic cardiomyopathy and acute idiopathic (Fiedler's) myocarditis: autopsy report. Intern Med 1993;32:815-9.

8. Sakai Y, Hayashi Y, Tomobuchi Y, et al. Dynamic outflow 
obstruction due to the transient extensive left ventricular wall motion abnormalities caused by acute myocarditis in a patient with hypertrophic cardiomyopathy: reduction in ventricular afterload by disopyramide. Jpn Circ J 1999;63:640-3.

9. Kusumoto S, Kawano H, Takeno M, et al. Cardiogenic shock due to left ventricular outflow obstruction and complete atrioventricular block in a patient with hypertrophic cardiomyopathy with acute myocarditis. Intern Med 2012;51:2565-71.

10. Frustaci A, Verardo R, Sale P, et al. Hypersensitivity myocarditis induced by beta-blockers: an unexpected cause of abrupt deterioration in hypertrophic cardiomyopathy. Intensive Care Med 2007;33:1848-9.

Cite this article as: Wang JY, Wang YS, Shen BT. Myocarditis combined with hypertrophic cardiomyopathy: a case report. Ann Palliat Med 2021;10(10):11203-11208. doi: 10.21037/apm-21359
11. Fisher DZ, DiSalvo TG, Dec GW, et al. Transient left ventricular aneurysm in a patient with hypertrophic cardiomyopathy and myocarditis. Clin Cardiol 1993;16:253-6.

12. Fujita S, Okamoto R, Takamura T, et al. Fulminant myocarditis in a patient with severe coronary artery disease. J Cardiol Cases 2013;9:15-7.

13. Burke MA, Cook SA, Seidman JG, et al. Clinical and Mechanistic Insights Into the Genetics of Cardiomyopathy. J Am Coll Cardiol 2016;68:2871-86.

14. Spillmann F, Kühl U, Van Linthout S, et al. Reversible transition from a hypertrophic to a dilated cardiomyopathy. ESC Heart Fail 2016;3:138-42. 